\title{
Prompt tau neutrinos at the LHC
}

\author{
Yu Seon Jeong* \\ Theoretical Physics Department, CERN, 1211 Geneva, Switzerland \\ E-mail: yuseon. jeong@cern.ch
}

\section{Weidong Bai}

Department of Physics and Astronomy, University of Iowa, Iowa City, IA 52242, USA

E-mail: weidong-bai@uiowa.edu

\section{Milind Diwan}

Brookhaven National Laboratory, USA

E-mail: diwan@bnl.gov

\section{Maria Vittoria Garzelli}

Universita' degli Studi di Firenze, Dipartimento di Fisica e Astronomia \& INFN, Firenze, Italy Eberhard Karls Universitaet Tuebingen, Institute For Theoretical Physics, Tuebingen, Germany

E-mail: garzelli@mi.infn.it

\section{Mary Hall Reno}

Department of Physics and Astronomy, University of Iowa, Iowa City, IA 52242, USA

E-mail: mary-hall-reno@uiowa.edu

\begin{abstract}
We investigate tau neutrinos from heavy flavor hadrons that can be explored at a high rapidity LHC experiment. A large number of tau neutrinos can be produced in $p p$ collision at the LHC in the very forward region, where its main source is $D_{s}^{ \pm}$mesons since the weak boson contribution is negligible. Abundant production of tau neutrinos will allow the precise study of tau neutrino charged current interactions to test lepton universality. In addition, it will provide the opportunity to probe the mixing between sterile neutrinos and tau neutrinos. We evaluate the fluxes and the event rates of the prompt tau neutrinos as well as the theoretical uncertainty using fits to the experimental data for charm meson production. Also, we describe the sterile neutrino masses and mixing angles that can be constrained by tau neutrinos from the LHC. ${ }^{\dagger}$
\end{abstract}

The 21st international workshop on neutrinos from accelerators (NuFact2019)

August 26 - August 31, 2019

Daegu, Korea

\footnotetext{
* Speaker.

$\dagger$ This work was supported in part by US-Department of Energy and the Korean Research Foundation (KRF) through the CERN-Korea Fellowship program. The authors would like to express a special thanks to the Mainz Institute for Theoretical Physics (MITP) of the Cluster of Excellence PRISMA+ (Project ID 39083149) for its hospitality and support.
} 


\section{Introduction}

In $p p$ collisions at the LHC, diverse hadrons are produced and decay to a number of neutrinos in the forward direction. While neutrinos are typically created from $\pi^{ \pm}$and $K^{ \pm}$, at sufficiently high energies, weak bosons and heavy flavor hadrons also contribute to the flux of neutrinos, which are referred to as prompt neutrinos. In the very large rapidity region $(y \gtrsim 6.5)$, the main source of prompt neutrinos is charm mesons, and in particular, tau neutrinos $\left(v_{\tau}\right)$ are mostly from the two body leptonic decays of the $D_{s}^{ \pm}$meson.

Interest in prompt $v_{\tau}$ detection has been increasing [1, 2, 3]. The FASER (ForwArd Search ExpeRiment) experiment at the LHC, the main goal of which is to search for weakly interacting BSM particles, would be able to detect neutrinos with the installation of additional neutrino detectors [4]. The FASER will set up a detector at $480 \mathrm{~m}$ from the ATLAS interaction point in the forward region, and it is planned in two stages. The approved first stage will collect data during the run 3 of the LHC for the pseudorapidity $(\eta)$ range of $\eta \gtrsim 9$, and the second stage is planning for the HL-LHC with a cylindrical detector, which has a radius consistent with $\eta \gtrsim 6.87$.

At such large rapidities, neutrino energy can be as high as a few $\mathrm{TeV}$, and thousands of $v_{\tau}$ events are expected in a modest sized detector [3,4], while the current world sample of clearly identified $v_{\tau}$ events is less than $10[5,6]$. Thus, detection of $v_{\tau}$ at the LHC will make it possible to investigate their charged current interaction more precisely and to test lepton universality. Moreover, the considerable flux and broad energy spectrum of $v_{\tau}$ will allow to explore the oscillation signals with sterile neutrinos $\left(v_{s}\right)$.

In this work, we evaluate the charm and beauty meson production at next-to-leading order (NLO) in perturbative QCD and compare the results with the LHCb data [7] to fit some parameters of theoretical calculation. Then, we estimate the fluxes and the event rates of prompt neutrinos focusing on $v_{\tau}$ for $\eta>6.87$. We also investigate the $v_{s}$ masses and mixing angles that can be probed by $v_{\tau}$ from the LHC.

\section{Heavy flavor production and decay to neutrinos}

The heavy quark production cross section can be evaluated using the parton model in perturbative QCD (pQCD). In the collinear approximation, initial state partons are assumed to be collinear with the beam, however, small transverse momentum $\left(k_{T}\right)$ effects can impact very forward heavy flavor production. A comparison with $\mathrm{LHCb}$ data [7] on $D_{s}^{ \pm}$production in the rapidity range $y=2.0-4.5$ is a testing ground for the incorporation of $k_{T}$ effects. One approach is to use POWHEG + PYTHIA8 [8], where NLO matrix elements for heavy-quark pair production are matched to the parton shower algorithms implemented in PYTHIA8. Alternatively, the effect of transverse momentum smearing can be incorporated by a 2-dimensional Gaussian function,

$$
f\left(\vec{k}_{T}\right)=\frac{1}{\pi\left\langle k_{T}^{2}\right\rangle} \exp \left[-k_{T}^{2} /\left\langle k_{T}^{2}\right\rangle\right] .
$$

The transverse momentum smearing can approximate effects of initial state showering and small intrinsic $k_{T}$ in event generators.

A comparison of the $D_{s}^{ \pm}$production cross section in NLO pQCD complemented by phenomenological fragmentation functions and the LHCb experimental data [7] is shown in the left 

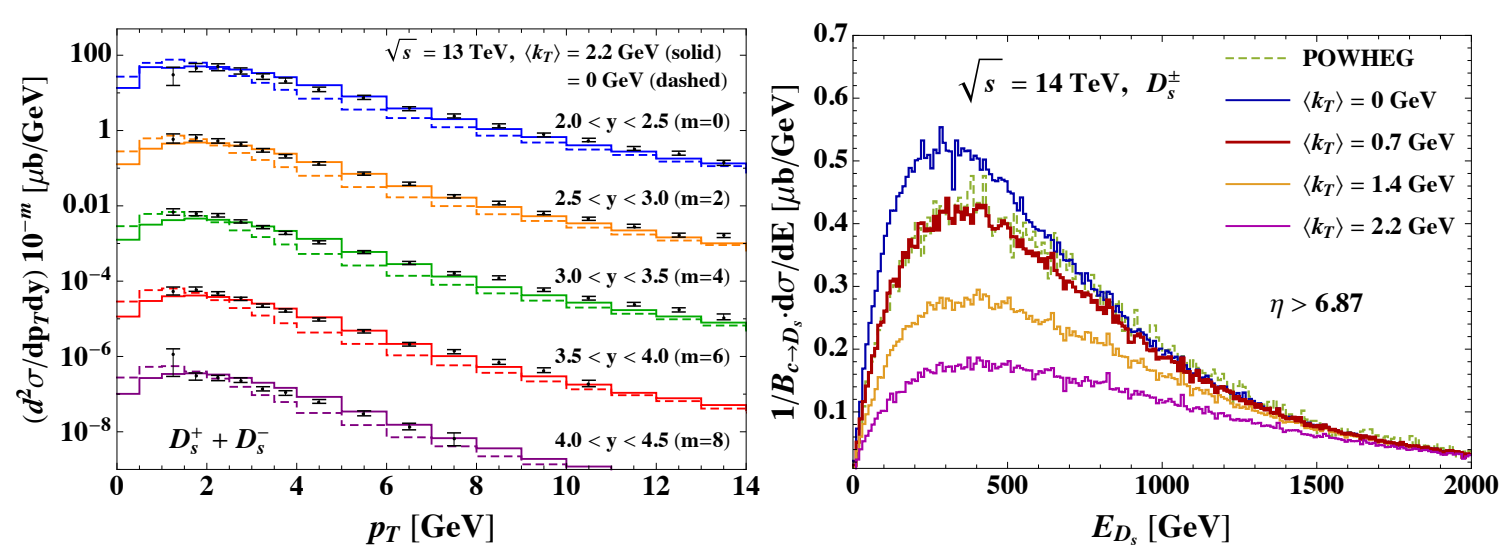

Figure 1: Left: The differential cross sections for $D_{s}^{+}+D_{s}^{-}$production in NLO pQCD and the corresponding LHCb data. The best fit with $\left\langle k_{T}\right\rangle=2.2 \mathrm{GeV}$ is presented with the results for $\left\langle k_{T}\right\rangle=0 \mathrm{GeV}$. Right: The differential cross section for $D_{s}^{+}+D_{s}^{-}$production in NLO pQCD as a function of $D_{s}$ energy for $\sqrt{s}=14 \mathrm{TeV}$ and $\eta>6.87$. A POWHEG + PYTHIA8 prediction is shown with the results for different values of $\left\langle k_{T}\right\rangle$.

panel of Fig. 1. By fitting to the data, we found the best fit as $\left\langle k_{T}\right\rangle=2.2 \mathrm{GeV}$ with $\chi^{2} /$ d.o.f $\simeq$ 2.8 for $\left(\mu_{R}, \mu_{F}\right)=\left(N_{R}, N_{F}\right) m_{T}=(1.0,1.5) m_{T}$ for the renormalization and factorization scales, respectively, where the transverse mass is defined as $m_{T}=\sqrt{m_{Q}^{2}+p_{T}^{2}}$ with $Q=c, b$. In the figure, the best fit predictions are shown with those in a collinear approximation $\left(\left\langle k_{T}\right\rangle=0 \mathrm{GeV}\right)$. The data show systematically higher cross sections at higher $p_{T}$ compared to the collinear approximation. This discrepancy requires further theoretical investigation.

The right panel of Fig. 1 presents $D_{s}$ production as a function of its energy for $\sqrt{s}=14 \mathrm{TeV}$ and $\eta>6.87$ for several values of $\left\langle k_{T}\right\rangle$ and from the POWHEG + PYTHIA8 simulation [8]. The latter agrees well with $\left\langle k_{T}\right\rangle=0.7 \mathrm{GeV}\left(\chi^{2} /\right.$ d.o.f $\left.\simeq 11.8\right)$ for Gaussian smearing, compatible with theoretical expectations for the $\left\langle k_{T}\right\rangle$ scale. We consider a range $\left\langle k_{T}\right\rangle=0-1.4 \mathrm{GeV}$ to estimate the uncertainty relative to theoretical expectations, and we comment on the impact of using the large best fit value of $\left\langle k_{T}\right\rangle=2.2 \mathrm{GeV}$.

Tau neutrinos are mainly from the decays of $D_{s}^{ \pm}$mesons; e.g.) $D_{s}^{+} \rightarrow \tau^{+}+v_{\tau}$ (direct decay) with $\operatorname{Br}\left(D_{s} \rightarrow \tau \nu_{\tau}\right) \sim 5.48 \%$ and the subsequent decay of the $\tau^{+}$to $\bar{v}_{\tau}$ (chain decay). Since most of $D_{s}^{+}$energy is transferred to the $\tau^{+}$in the direct decay, $\bar{v}_{\tau}$ from the chain decay are dominant at high energies. The total $v_{\tau}$ and $\bar{v}_{\tau}$ distributions are equal. This is reflected in the left plot of Fig. 2, which shows the differential cross section for $v_{\tau}$ and $\bar{v}_{\tau}$ production for the direct decay, the chain decay and the total neutrinos from both channels. When multiplied by the luminosity, the curve in the plot produces the total flux of neutrinos plus anitneutrinos incident on the whole detector solid angle per unit energy. We also take into account $B$ meson contributions to $v_{\tau}$. Compared with $D_{s}^{ \pm}$ contribution, neutrinos from the $B^{ \pm}, B^{0}$ and $\bar{B}^{0}$ mesons are less than those from $D_{s}^{ \pm}$by $\sim 1-2$ orders of magnitude over the energy range of $0.5-2 \mathrm{TeV}$.

The right panel of Fig. 2 shows the event rates of $v_{\tau}$ and $\bar{v}_{\tau}$ from $D_{s}^{ \pm}$evaluated for $2 \mathrm{~m}$ of lead target with $\mathscr{L}=3 \mathrm{ab}^{-1}$ with no experimental efficiencies included. The central value is for the value of $\left\langle k_{T}\right\rangle=0.7 \mathrm{GeV}$ and the bands indicate the uncertainty by $\left\langle k_{T}\right\rangle$ from $0-1.4 \mathrm{GeV}$. For total event numbers of $v_{\tau}$ and $\bar{v}_{\tau}$, we obtain $7568(6797,7886)$ for $\left\langle k_{T}\right\rangle=0.7(1.4,0) \mathrm{GeV}$ with $\sim$ $4 \%$ contribution from the $B$ mesons. Using $\left\langle k_{T}\right\rangle=2.2 \mathrm{GeV}$, the total number of events is 5724 . 

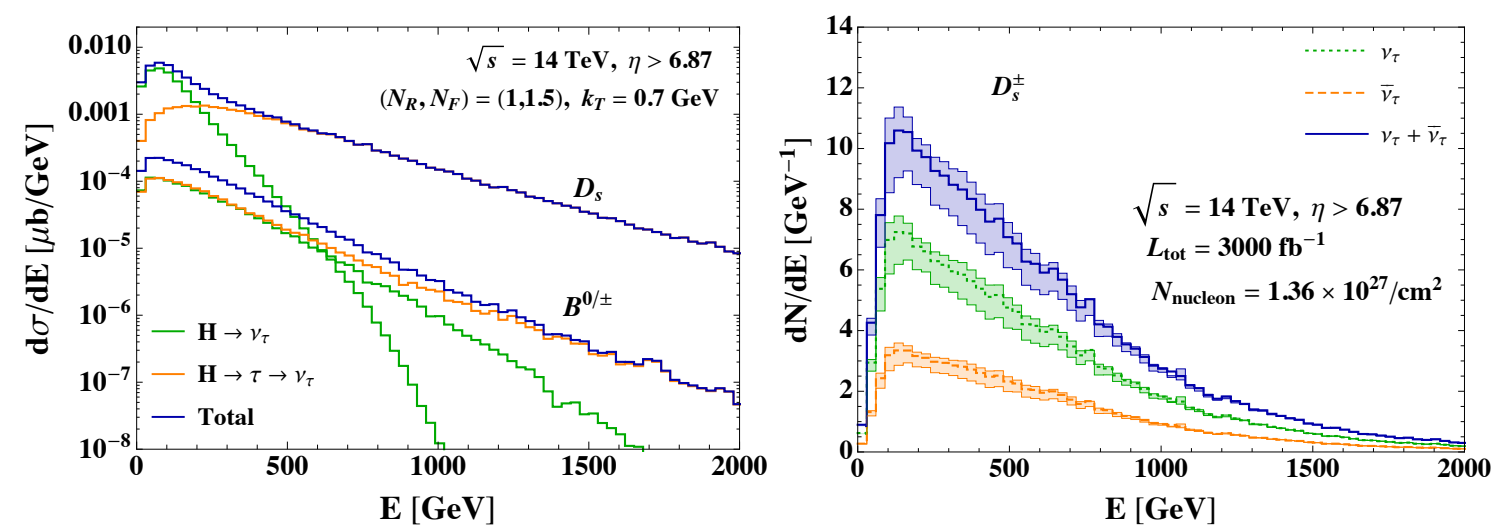

Figure 2: Left: The differential cross section for $v_{\tau}+\bar{v}_{\tau}$ from the direct decay $\left(H \rightarrow v_{\tau}\right)$ and the chain decay $\left(H \rightarrow \tau \rightarrow v_{\tau}\right)$ with $H=D_{s}^{ \pm}, B^{0, \pm}$ and $\bar{B}^{0}$. Right: The estimation of $v_{\tau}$ and/or $\bar{v}_{\tau}$ event rates from the decays of $D_{s}$ mesons. The central result is for the value of $\left\langle k_{T}\right\rangle=0.7 \mathrm{GeV}$ and the shaded corresponds to the uncertainty in $\left\langle k_{T}\right\rangle$ range of $0-1.4 \mathrm{GeV}$.

\section{Oscillation with sterile neutrinos}

Sterile neutrinos are searched for over a very wide mass range with different motivations. The accelerator and reactor oscillation experiments mainly probe $\mathrm{eV}$-scale $v_{s}$ motivated by the SBL anomaly $[9,10]$. Due to a considerable number of $v_{\tau}$ and their broad energy spectrum, $v_{\tau}$ oscillations in the presence of $v_{s}$ can be investigated with $v_{\tau}$ produced in the very forward region at the LHC. Since the baseline and neutrino energy range are not sensitive to oscillation between the SM neutrinos, distortions in the event spectrum can be interpreted as oscillation signals with $v_{s}$.

Oscillation probabilities in the two flavor approximation in a $3+1$ model are given by

$$
P\left(v_{\alpha} \rightarrow v_{\beta}\right) \simeq \delta_{\alpha \beta} \mp \sin ^{2} 2 \theta_{\alpha \beta} \sin ^{2}\left(\Delta m_{s}^{2} L / 4 E\right) \quad(+ \text { for } \alpha \neq \beta),
$$

where $\sin ^{2} \theta_{\alpha \beta}=4\left(1-\left|U_{\alpha 4}\right|^{2}\right)\left|U_{\alpha 4}\right|^{2}$ for $\alpha=\beta$ and $4\left|U_{\alpha 4}\right|^{2}\left|U_{\beta 4}\right|^{2}$ for $\alpha \neq \beta$. For optimal signal in the oscillation experiments, the following condition should be satisfied,

$$
\frac{\Delta m_{s}^{2} L}{4 E}=\frac{\pi}{2}
$$

As shown in Fig. 2, the energy at the maximum event rates is near $200 \mathrm{GeV}$, where a significant effect can be observed if there is oscillation with $v_{s}$. This energy and the distance to the detector $L=480 \mathrm{~m}$ yield $\Delta m_{s}^{2} \sim 500 \mathrm{eV}^{2}$, equivalent to $m_{s} \sim 20 \mathrm{eV}$. For mixing parameters, there exist only a few constraints for $\Delta m_{s}^{2}>100 \mathrm{eV}^{2}[11,12]$. Using the strongest constraints from NOMAD, we choose two testable parameter sets as

$$
\left(\left|U_{e 4}\right|^{2},\left|U_{\mu 4}\right|^{2},\left|U_{\tau 4}\right|^{2}\right)=\left(0.04,10^{-3}, 0.08\right) \text { and }\left(0.02,5 \times 10^{-4}, 0.15\right)
$$

with $m_{s}=20 \mathrm{eV}$. The expected event rate spectra affected by disappearance of $v_{\tau}$ and appearance from $v_{\mu / e} \rightarrow v_{\tau}$ for the parameters in Eq. 3.3 are obtained as shown in Fig. 3. As in Fig. 2, only detector geometry is considered in evaluations. Oscillations of $v_{e} \rightarrow v_{\tau}$ increase the $v_{\tau}$ event rates by $\sim 15 \%$ at $E \sim 200 \mathrm{GeV}$ for both of the parameter sets, which partially offsets the reduced $v_{\tau}$ event rates due to $v_{\tau} \rightarrow v_{e / \mu / s}$. The oscillations of $v_{\mu} \rightarrow v_{\tau}$ have an impact of less than $1 \%$. 


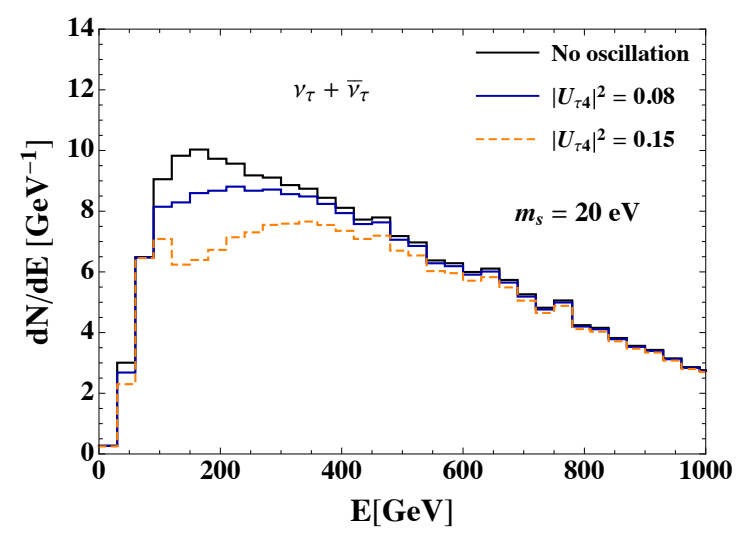

Figure 3: The predictions of $v_{\tau}+\bar{v}_{\tau}$ event rates in the SM and in a model with a $v_{s}$. See text for more details.

\section{Summary}

We have investigated tau neutrinos produced at the LHC in the very forward region for $\eta \gtrsim$ 6.87 (for more complete investigations, see Ref. [13]). Experiments to measure these neutrinos will provide an opportunity for the first detection of neutrinos from a collider with the highest energy neutrinos from a terrestrial experiment. The expected number of tau neutrino events are several thousand per $\mathrm{m}$ of the detector length and their energies will be as high as a few TeV. Due to an abundant number of events, detection of these tau neutrinos will contribute to the precise study of tau neutrino interaction up to a few $\mathrm{TeV}$ and of heavy flavor production in the forward region. In addition, it will be an opportunity to explore $v_{s}$ of larger masses than usually investigated.

\section{References}

[1] M. Anelli et al. [SHiP Collaboration], arXiv:1504.04956; S. Alekhin et al., Rept. Prog. Phys. 79, no. 12, 124201 (2016).

[2] S. Aoki et al. [DsTau Collaboration], arXiv:1906.03487.

[3] N. Beni et al., J. Phys. G 46, no. 11, 115008 (2019); [XSEN Collaboration], arXiv:1910.11340.

[4] A. Ariga et al. [FASER Collaboration], arXiv:1901.04468; H. Abreu et al. arXiv:1908.02310.

[5] K. Kodama et al. [DONUT Collaboration], Phys. Rev. D 78, 052002 (2008).

[6] N. Agafonova et al. [OPERA Collaboration], Phys. Rev. Lett. 120, no. 21, 211801 (2018) Erratum: [Phys. Rev. Lett. 121, no. 13, 139901 (2018)].

[7] R. Aaij et al. [LHCb Collaboration], JHEP 1603, 159 (2016) Erratum: [JHEP 1705, 074 (2017)].

[8] S. Alioli, P. Nason, C. Oleari and E. Re, JHEP 1006, 043 (2010).

[9] A. Aguilar-Arevalo et al. [LSND Collaboration], Phys. Rev. D 64, 112007 (2001); [MiniBooNE Collaboration], Phys. Rev. Lett. 98, 231801 (2007); Phys. Rev. Lett. 121, no. 22, 221801 (2018).

[10] G. Mention et al. Phys. Rev. D 83, 073006 (2011).

[11] K. Abe et al. [Super-Kamiokande Collaboration], Phys. Rev. D 91, 052019 (2015); E. Eskut et al. [CHORUS Collaboration], Nucl. Phys. B 793, 326 (2008).

[12] P. Astier et al. [NOMAD Collaboration], Nucl. Phys. B 611, 3 (2001); P. Astier et al. [NOMAD Collaboration], Phys. Lett. B 570, 19 (2003).

[13] W. Bai, M. Diwan, M. V. Garzelli, Y. S. Jeong and M. H. Reno, arXiv:2002.03012 [hep-ph]. 